\title{
Effects of activation of AMPK on human breast cancer cell lines with different genetic backgrounds
}

\author{
OMAR S. EL-MASRY, BARRY L. BROWN and PAULINE R.M. DOBSON \\ Department of Molecular Biology and Biotechnology, University of Sheffield, Western Bank, Sheffield S10 2TN, UK
}

Received May 26, 2011; Accepted October 4, 2011

DOI: $10.3892 / 01.2011 .458$

\begin{abstract}
Breast cancer remains a therapeutic challenge, and this has intensified the search for new drug targets. The AMP-activated protein kinase (AMPK) signalling pathway is emerging as having potential for intervention. We assessed the possible different effects of AMPK action on breast cancer cells by studying their impact on proliferation, apoptosis and the mitochondrial membrane potential in three breast cancer cell lines (MCF-7, MDA-MB-231 and T47D) differing in their p53 and estrogen receptor (ER) status. Activation of AMPK by 5-aminoimidazole carboxamide ribonucleotide (AICAR) and phenformin elicited clear anti-proliferative effects in all breast cancer cell lines, but with differences in sensitivity. However, the anti-proliferative effects were accompanied by varying responses between the different cells, with a marked cell cycle arrest effect in T47D cells and an apoptotic effect in MCF-7 and MDA-MB-231 cells. The mitochondrial apoptotic pathway was potentially involved in all cell lines. These results suggest that AMPK potentially serves as a therapeutic target in breast cancer, but one which may be dependent on the genetic background of the cancer cells.
\end{abstract}

\section{Introduction}

Breast cancer has a high incidence worldwide (1). Factors that may play a role in the onset and progression of breast cancer include genetic, metabolic and lifestyle-associated risk factors. Obesity is a risk factor via its role in increasing circulating estrogen, insulin, insulin-like growth factor and adipokines, all of which may contribute to cancer initiation and progression (2). Mutations in certain tumour suppressor genes also contribute to risk of cancer (3). One such tumour suppressor is LKB1, which is found to be mutated in Peutz-Jegher syndrome and a number of other types of cancer, including certain types of breast cancer (4). LKB1 is a serine/threonine kinase that

Correspondence to: Dr Pauline Dobson, Department of Molecular Biology and Biotechnology, University of Sheffield, Western Bank, Sheffield, S10 2TN, UK

E-mail: p.dobson@sheffield.ac.uk

Key words: breast cancer, AMP-activated protein kinase phosphorylates downstream substrates, such as AMP-activated protein kinase (AMPK) and related kinases (5). AMPK is a master metabolic regulator of energy in all cells and structurally is a trimeric enzyme comprising 3 subunits, $\alpha, \beta$, and $\gamma$. The catalytic activity belongs to the $\alpha$ subunit, while the regulatory functions belong to the other two subunits. AMPK is activated in response to phosphorylation of the critical amino acid residue Thr172. This phosphorylation process is catalysed by a number of upstream kinases including LKB1 and calmodulin-dependent kinase kinase (CaMKK) (6).

The ability of LKB1 to phosphorylate and activate AMPK, and, moreover, the observation that absence of LKB1 in certain cell types is accompanied by a lack of AMPK activity, indicates that the action of LKB1 as a tumour suppressor is attributed, at least in part, to AMPK (5). This assessment was supported and consolidated by a number of observations. AMPK was reported to activate and phosphorylate the trans-active domain of p53 (7) and p21 (8), disrupting the cell cycle. AMPK has also been shown to inhibit mTOR and, thereby, protein biosynthesis. AMPK enhances the formation of the tuberous sclerosis complex 1 (TSC1)/tuberous sclerosis complex 2 (TSC2) complex which dephosphorylates and inactivates Rheb, an upstream activator of mTOR (9). Lipogenic enzymes, fatty acid synthase (FA) and acetyl CoA carboxylase (ACC), are highly expressed in cancer cells as a result of the high demand for fatty acids that are required to be incorporated in the plasma membranes of the newly dividing cells. The two enzymes were suppressed by AMPK activation in cancer cells (10).

There have been reports of apparent anti-cancer effects of AMPK activators in breast cancer cells (11). The present study consolidates and expands on these observations and describes the results of investigations on the effect of AMPK activation on cell proliferation and apoptosis in three breast cancer cell types differing in their estrogen receptor (ER) and p53 status.

\section{Materials and methods}

Materials. 5-aminoimidazole carboxamide ribonucleotide (AICAR) and phenformin were obtained from Sigma-Aldrich (Poole, Dorset, UK). Dulbecco's minimal essential medium (DMEM) was obtained from Gibco BRL (Paisley, UK) and foetal calf serum (FCS) from Autogenbioclean (UK Ltd.). Isoton was from Beckman Coulter UK, Ltd. (High Wycombe, $\mathrm{UK}$ ), phosphate-buffered saline (PBS) and trypsin/EDTA were obtained from Sigma-Aldrich (Irvine, Ayrshire, UK), 
Cell titer $96^{\circledR}$ Aqueous One Solution cell proliferation assay was from Promega (Madison, WI, USA), MitoProbe ${ }^{\mathrm{TM}}$ JC-1 assay kit for flow cytometry was from Invitrogen (Paisley, UK) and Mitocasp ${ }^{\mathrm{TM}}$ (S-9101 Caspase 3/7) was from Bachem (San Carlos, CA, USA). T25 and T75 tissue culture flasks were from Nunc ${ }^{\mathrm{TM}}$ (Denmark) and tissue culture sterile 6-, 24- and 96-well plates from Costar ${ }^{\circledR}$, USA. Blotting reagents were purchased from BioRad (CA, USA and München, Germany), with the exception of TBST and the transfer buffer, which were prepared from their components.

Breast cancer cell lines with different genetic backgrounds regarding p53 and ER were used to investigate the anti-cancer effects of AMPK. The breast cancer cell lines MCF-7 ( $553^{+}$ and $\mathrm{ER}^{+}$), T47D (p53 mutant and $\mathrm{ER}^{+}$) and MDA-MB-231 (p53 mutant and $\mathrm{ER}^{-}$) were purchased from ECACC (Porton Down, UK).

Cell culture. Breast cancer cell lines were grown in a continuous monolayer culture in T75 top filtered sterile tissue culture flasks inside a sterile humidified incubator at $37^{\circ} \mathrm{C}$ with $5 \%$ $\mathrm{CO}_{2}$ in air. Cells were then sub-cultured as required for maintenance and to plate cells for experimental work.

Western blotting. Cell lines were sub-cultured, counted and plated at $1 \times 10^{6}$ cells in appropriate tissue culture plates and incubated at $37^{\circ} \mathrm{C}$ with $5 \% \mathrm{CO}_{2}$ in air. Cells were then incubated with $\operatorname{AICAR}(0.83 \mathrm{mM})$ or phenformin $(2 \mathrm{mM})$ with corresponding controls for $1 \mathrm{~h}$. Cell lysates were collected from the control and treated cells, followed by a protein assay. Equal protein concentrations of control-, AICAR- and phenformin-treated samples were loaded at a maximum volume of $30 \mu \mathrm{l} /$ well into pre-formed tris-HCL-SDS gels (BioRad). Proteins were then separated by electrophoresis and transferred to a PVDF membrane. The membrane was then blocked overnight in $10 \%$ milk prior to being incubated overnight at $4^{\circ} \mathrm{C}$ with anti-phospho AMPK antibody (Cell Signaling Technology, Danvers, MA, USA) directed against the crucial Thr172 amino acid residue. The membrane was washed in TBST four times for 15 min each and incubated with polyclonal anti-rabbit IgG antibody (Cell Signaling Technology, \#7074) and then washed again four times prior to the addition of the fluorescence substrate. Band density was then estimated using imaging system tools (Syngene, G: Box, Geneflow, UK).

Cell proliferation. Cells were sub-cultured, counted by the Coulter cell counter (Coulter Electronics ZI, Luton, Bedfordshire, UK), plated at $7 \times 10^{3}$ cells/well in sterile 96 -well plates and incubated overnight at $37^{\circ} \mathrm{C}$ with $5 \% \mathrm{CO}_{2}$ in air. The following day, serial concentrations of AICAR $(0-1.66 \mathrm{mM})$ or phenformin (0-4 mM) were added. The number of viable cells was then estimated following $72 \mathrm{~h}$ of incubation via their metabolic activity using the Aqueous One assay kit (Promega), which assesses the ability of cells to reduce the colourless tetrazolium, producing red-coloured formazan, whose colour was measured at 490 and $630 \mathrm{~nm}$ using a Biotek ELX800 Microplate reader (Biotek Instruments, Winooski, VT, USA).

Microscopy. The cell lines were treated with $0.83 \mathrm{mM}$ AICAR or $2 \mathrm{mM}$ phenformin after being sub-cultured, counted and plated at $2 \times 10^{5}$ cells/well in sterile tissue culture 6-well plates. Treated cells and corresponding controls were then examined for morphological features of apoptosis following incubation periods of 24, 48 and $72 \mathrm{~h}$ under the Xli-Cap software linked light microscope (Xli, CETi, UK). Images were collected after $24 \mathrm{~h}$ for control-, AICAR- and phenformin-treated cells, followed by re-incubation in the same culture conditions, the process being repeated after 48 and $72 \mathrm{~h}$ of incubation.

Effect of AMPK activation on mitochondrial membrane potential. Mitochondrial membrane potential was assessed using a fluorescence activated cell sorter (FACSCalibur, BD Biosciences, NJ, USA). The principle involves the use of a dye (JC-1) that accumulates inside mitochondria with intact membrane potential, producing a red fluorescence. Depolarisation results in leakage of the dye with a concomitant decrease in red fluorescence.

Breast cancer cell lines were sub-cultured, counted and plated at $2 \times 10^{5}$ cells/well in sterile 6-well tissue culture plates and incubated overnight at $37^{\circ} \mathrm{C}$ with $5 \% \mathrm{CO}_{2}$. The medium was removed and the cells were then washed and treated with AICAR $(0.83 \mathrm{mM})$ or phenformin $(2 \mathrm{mM})$ and incubated with corresponding controls in the same culture conditions for $24 \mathrm{~h}$. The cell monolayer was then washed with PBS (retaining the detached cells) followed by the release of the monolayer by trypsin/EDTA. The detached cells were then combined with their corresponding released cells and pelleted by centrifugation at $200 \mathrm{x} \mathrm{g}$ at $4^{\circ} \mathrm{C}$ for $10 \mathrm{~min}$. Cell pellets were then washed twice in the provided washing buffer by being subjected to centrifugation at $200 \mathrm{x}$ g at $4^{\circ} \mathrm{C}$ for $10 \mathrm{~min}$. The cells were then re-suspended in $300 \mu \mathrm{l}$ washing buffer prior to the addition of $10 \mu 1$ of fluorochrome. Labelled cells were then incubated at $37^{\circ} \mathrm{C}$ with $5 \% \mathrm{CO}_{2}$ for $30-60 \mathrm{~min}$ until use for flow cytometric analysis with appropriate filters.

\section{Results}

Western blotting. To confirm that AICAR and phenformin activated AMPK in these cell lines, the effect of the two agents on the phosphorylation of the enzyme by Western blotting was investigated. The immunoblotting results revealed that AMPK in treated cells was more phosphorylated than untreated control cells (Fig. 1A-C). No discernable difference was evident in the total AMPK between each of the cell lines (data not shown).

Cell proliferation. Increasing doses of the AMPK activator (AICAR) resulted in dose responsive decreases in the proliferation of all 3 breast cancer cell lines (Fig. 2A). T47D cells appeared to be the most sensitive to the action of AICAR, whereas the MDA-MB-231 cells were least sensitive. Phenformin also inhibited proliferation of the breast cancer cells, the effects being similar in all three cell types (Fig. 2B). The maximum effect of phenformin was greater than that of AICAR, which may be attributed, at least in part, to its high lipid/water partition coefficient over AICAR.

Microscopy. Over time, AMPK activation by AICAR or phenformin induced typical apoptotic morphological features including membrane blebbing and eventually fragmentation of cells into apoptotic bodies in MCF-7, T47D and MDA-MB-231 
A

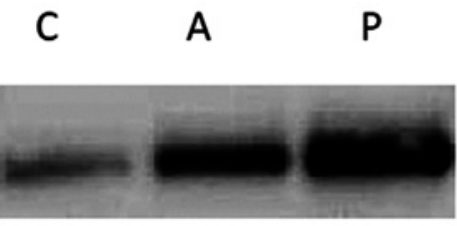

MCF-7 cells

B

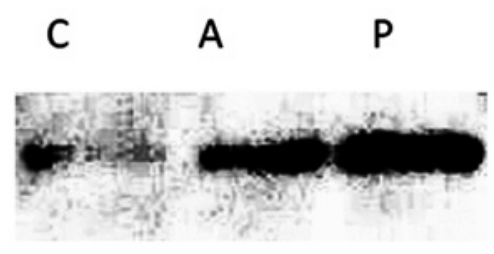

MDA-MB-231 cells

C

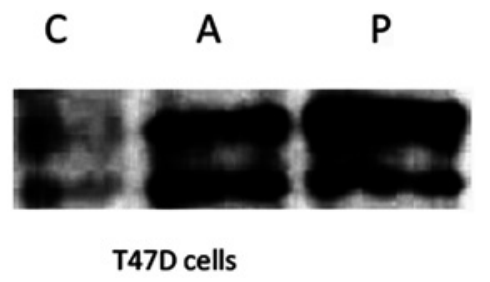

Figure 1. Western blotting results of phospho-AMPK and total AMPK in the control-, AICAR-treated cells. (A) MCF-7, (B) MDA-MB-231 and (C) T47D cells. (C, control; A, AICAR; P, phenformin).

A

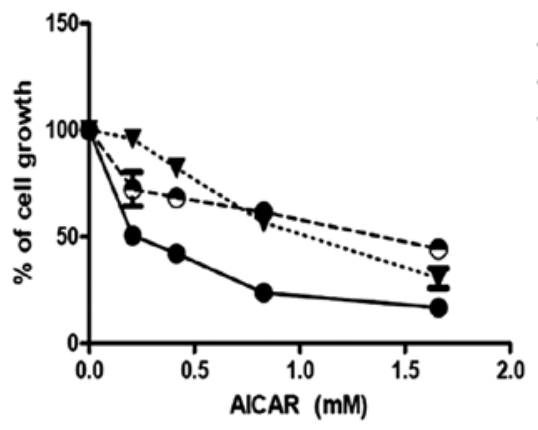

B

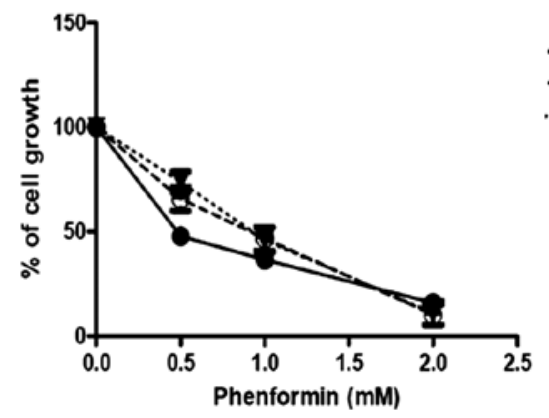

Figure 2. Effect of increasing doses of (A) AICAR and (B) phenformin on breast cancer cell proliferation.

cells (data not shown). It was clear that the effect of AICAR was much slower than that of phenformin. This slower effect of AICAR may be due to the different pharmacokinetic properties between AICAR and phenformin, as it is less lipophilic.

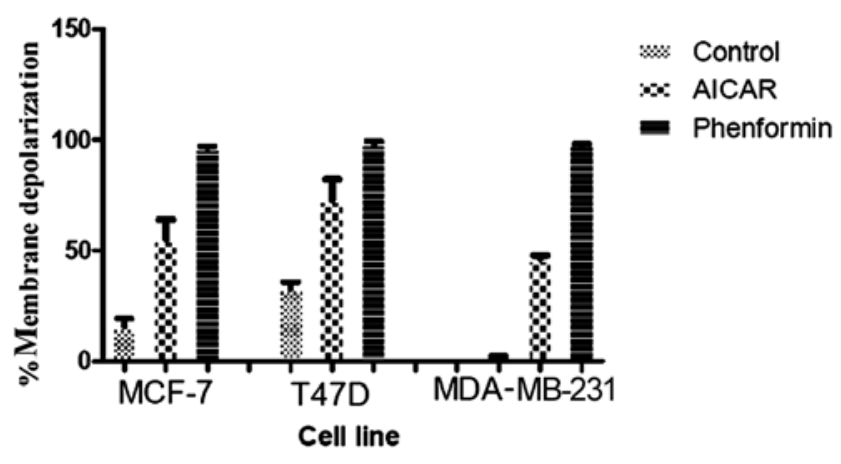

Figure 3.Effect of AMPK activation by AICAR and phenformin on the mitochondrial membrane potential in MCF-7, MDA-MB-231 and T47D breast cancer cell lines.

Of note, the apoptotic response to AMPK activation was lower in T47D cells than in the MCF-7 and MDA-MB-231 cell lines, with T47D cells having the highest FasL expression.

Mitochondrial membrane potential. The activation of AMPK was found to induce mitochondrial membrane depolarisation in all three cell types (Fig. 3). While phenformin resulted in almost complete depolarisation, the effect of AICAR was sub-maximal. Notably, AMPK activation by AICAR and phenformin disrupted mitochondrial membrane potential with a significant difference between control- and AICARor phenformin-treated cells; MCF-7 (control AICAR, $\mathrm{p}=0.016$ and control phenformin, $\mathrm{p}=0.0001$ ), T47D (control AICAR, $\mathrm{p}=0.015$ and control phenformin, $\mathrm{p}<0.0001)$ and MDA-MB-231 (control AICAR, $\mathrm{p}<0.0001$ and control phenformin, $\mathrm{p}<0.0001)$. In addition, we have confirmed the finding of Yang et al (12) that basal caspase 3 and/or 7 activity has been detected in breast cancer cell lines in the absence of any apoptotic stimuli, as indicated by the presence of activated caspases 3 and 7 in the control as well as AICAR- and phenformin-treated cells.

\section{Discussion}

It is now clear that AMPK is not just a family of kinases involved in metabolic regulation by switching on and off metabolic pathways to control the energy status inside cells. Although not enough is known about the complete roles of AMPK, it is realised that AMPK is a multifunctional regulatory protein whose functions involve central (13) and peripheral regulation. It was shown that activation of AMPK steered three breast cancer cell lines with differing genetic backgrounds towards cell death and functioned as an anti-proliferation agent. This anti-proliferative effect of AMPK activation has been reported to be via its ability to activate p53 (7) and p21 (8), thereby interfering with the cell cycle. Although numerous studies have involved p53 in the pro-apoptotic action of AMPK, we report a similar effect in a breast cancer cell line, MDA-MB-231, that lacks wild-type p53. This suggests a p53-independent pro-apoptotic action. In the present study, T47D cells (also lacking wild-type p53) were the most sensitive to the effect of AICAR. However, in T47D cells, the effect appeared to be via cell cycle arrest more than via apoptosis. 
In other studies, AICAR had a cytotoxic effect on acute lymphoblastic leukemia cells, an action that was correlated with AMPK activation (14). A notable finding was that AMPK activation had an anti-apoptotic effect in normal brain cells, whereas, it was pro-apoptotic in tumour brain cells (15).

Three main apoptotic pathways have been identified thus far: extrinsic; intrinsic or mitochondrial; and the T cell-mediated pathways. All pathways require involvement of certain aspartate proteases that catalyse limited proteolysis of proteins at aspartic acid and are collectively known as caspases. Caspases are either initiators (caspase 2,8,9 and 10) or executioners (also called effectors; caspase 3, 6 and 7). The mitochondrial or intrinsic pathway requires the release of cytochrome $\mathrm{C}$ from the mitochondria, an event that requires a prior disruption of the mitochondrial membrane potential. This event is followed by activation of initiator caspases that ultimately activate the executioner caspases 3 and 7 in a cascade of limited proteolysis (16).

In the present study, the mitochondrial or intrinsic apoptotic pathway was found to be another target for AMPK. We observed that in the breast cancer cell lines (with and without p53 or ER), AMPK activation resulted in disruption of the mitochondrial membrane potential. These results indicate that activation of AMPK potentially induces apoptosis via a p53 or p21-independent pathway. It has been reported that breast cancer cell lines have high basal levels of caspase activation, which aid in cell migration and metastasis, completely independently of any apoptotic stimuli (12).

Mitochondrial respiratory complex 1 has been reported to be a target of metformin and thialozidinediones (TZDs) that are widely used to treat diabetic patients suffering from severe insulin resistance. Inhibition by metformin of the mitochondrial respiratory complex 1 and the resultant impairment of mitochondrial function and cellular respiration has been thought to be responsible for its anti-diabetic effect. The mechanism of action of metformin was not clear until the report (17) that showed that AMPK mediates metformin action, and that inhibition of AMPK eliminated the effect of metformin. This mechanism indicates that the mitochondrial membrane potential may be affected by activated AMPK and trigger the intrinsic pathway of apoptosis by enhancing the release of the pro-apoptotic factors from mitochondria. This action is supported by our study on three breast cancer cell lines differing in their genetics regarding p53 and ER status.

The enhanced effect of phenformin as a mitochondrial membrane potential disruptor may be attributed to the previously reported affinity of biguanides, including phenformin, to accumulate inside mitochondria, an action that has not been reported for AICAR. These observations suggest that the effect of AICAR on the mitochondrial membrane potential may be mediated by AMPK. However, the effect is slower than that of phenformin due to differences in their pharmacokinetic properties. The effect of phenformin could be direct or indirect via an ATP decrease-dependent activation of AMPK.

MDA-MB-231 cells were the least sensitive in most assessments; however, AMPK activation in MDA-MB-231 cells induced mitochondrial membrane potential disruption to the same degree as that noted in other cell lines. It is unlikely that the p53 status of the MDA-MB-231 cells is responsible for their low sensitivity to AMPK-induced growth inhibition, since the most sensitive cells, T47D, also express mutant $\mathrm{p} 53$. However, further investigations are required to delineate the underlying reasons. In addition, T47D cells were the least sensitive to apoptotic changes, as was observed in photomicrographs. Unlike MCF-7 and MDA-MB-231 cells, the cell cycle analysis of T47D cells revealed a cell cycle arrest effect of AMPK activation by AICAR and phenformin rather than the apoptotic effect which was more apparent in the MCF-7 and MDA-MB-231 cell lines.

The greater sensitivity of T47D cells may be related to the fact that Western blotting revealed two bands reacting to the phospho-AMPK antibody, whereas only one band was observed in lysates from MCF-7 and MDA-MB-231 cells.

In conclusion, we have observed significantly different effects of AMPK activation on the growth and death of three human breast cancer cell lines. Thus far, it is not possible to ascribe these differences to p53 status or ER expression, which are key differences that have been reported previously. However, further studies are required to ascertain the intracellular signalling pathways responsible, and the possible interactions (crosstalk) with other signals. Furthermore, the extent to which AMPK may be a target or co-target for therapeutic intervention in breast cancer should be explored.

\section{References}

1. Evans DG and Howell A: Breast cancer risk-assessment models: Breast Cancer Res 9: 213, 2007.

2. Lorincz AM and Sukumar S: Molecular links between obesity and breast cancer. Endocr Relat Cancer 13: 279-292, 2006.

3. Todorov PT, McDevitt TM, Meyer DJ, Ueyama H, Ohkubo I and Tisdale MJ: Purification and characterization of a tumor lipid-mobilizing factor. Cancer Res 58: 2353-2358, 1998.

4. Katajisto P, Vallenius T, Vaahtomeri K, Ekman N, Udd L, Tiainen M and Makela TP: The LKB1 tumor suppressor kinase in human disease. Biochim Biophys Acta 1775: 63-75, 2007.

5. Lizcano JM, Goransson O, Toth R, Deak M, Morrice NA, Boudeau J, Hawley SA, Udd L, Makela TP, Hardie DG and Alessi DR: LKB1 is a master kinase that activates 13 kinases of the AMPK subfamily, including MARK/PAR-1. EMBO J 23: 833-843, 2004

6. Viollet B, Athea Y, Mounier R, Guigas B, Zarrinpashneh E, Horman S, Lantier L, Hebrard S, Devin-Leclerc J, Beauloye C, Foretz M, Andreelli F, Ventura-Clapier R and Bertrand L: AMPK: Lessons from transgenic and knockout animals. Front Biosci 14: 19-44, 2009.

7. Bode AM and Dong Z: Post-translational modification of p53 in tumorigenesis. Nat Rev Cancer 4: 793-805, 2004.

8. Caraci F, Chisari M, Frasca G, Chiechio S, Salomone S, Pinto A, Sortino MA and Bianchi A: Effects of phenformin on the proliferation of human tumor cell lines. Life Sci 74: 643-650, 2003

9. Luo Z, Saha AK, Xiang X and Ruderman NB: AMPK, the metabolic syndrome and cancer. Trends Pharmacol Sci 26: 69-76, 2005.

10. Xiang X, Saha AK, Wen R, Ruderman NB and Luo Z: AMP-activated protein kinase activators can inhibit the growth of prostate cancer cells by multiple mechanisms. Biochem Biophys Res Commun 321: 161-167, 2004.

11. Rattan R, Giri S, Singh AK and Singh I: 5-Aminoimidazole4-carboxamide-1-beta-D-ribofuranoside inhibits cancer cell proliferation in vitro and in vivo via AMP-activated protein kinase. J Biol Chem 280: 39582-39593, 2005.

12. Yang L, Cao Z, Yan H and Wood WC: Coexistence of high levels of apoptotic signaling and inhibitor of apoptosis proteins in human tumor cells: implication for cancer specific therapy. Cancer Res 63: 6815-6824, 2003.

13. Butler $\mathrm{H}$ and Korbonits M: Cannabinoids for clinicians: the rise and fall of the cannabinoid antagonists. Eur J Endocrinol 161: 655-662, 2009. 
14. Sengupta TK, Leclerc GM, Hsieh-Kinser TT, Leclerc GJ, Singh I and Barredo JC: Cytotoxic effect of 5-aminoimidazole-4-carboxamide-1-beta-4-ribofuranoside (AICAR) on childhood acute lymphoblastic leukemia (ALL) cells: implication for targeted therapy. Mol Cancer 6: 46, 2007.

15. Mukherjee P, Mulrooney TJ, Marsh J, Blair D, Chiles TC and Seyfried TN: Differential effects of energy stress on AMPK phosphorylation and apoptosis in experimental brain tumor and normal brain. Mol Cancer 7: 37, 2008.
16. Elmore S: Apoptosis: a review of programmed cell death. Toxicol Pathol 35: 495-516, 2007.

17. Zhou G, Myers R, Li Y, Chen Y, Shen X, Fenyk-Melody J, Wu M, Ventre J, Doebber T, Fujii N, Musi N, Hirshman MF, Goodyear LJ and Moller DE: Role of AMP-activated protein kinase in mechanism of metformin action. J Clin Invest 108: 1167-1174, 2001. 